\title{
LI. On polarizing prisms
}

\section{R.T. Glazebrook M.A. F.R.S.}

To cite this article: R.T. Glazebrook M.A. F.R.S. (1883) LI. On polarizing prisms, Philosophical Magazine Series 5, 15:95, 352-362, DOI: 10.1080/14786448308627362

To link to this article: http://dx.doi.org/10.1080/14786448308627362

曲 Published online: 28 Apr 2009.

6 Submit your article to this journal $\pi$

Џll Article views: 2

Q View related articles $₫$ 
probably varies as the pure resistance varies, and further remembering that an opposing electromotive force which has no existence apart from a combined resistance acts in any electrical test exactly as a resistance, it must be always very difficult experimentally to separate them. All of course that wo can measure electrically is the difference of potential between the carbons and the current passing between them ; and this is what wo have been measuring all through these two investigations.

It may be here noted that in all probability the conduction from particle to particle in a microphone is of the nature of $a$ small electric arc, or, rather, perhaps a convective discharge, seeing that the resistance in a microphone varies with the current used to measure it; indeed it is probable, when the pieces of carbon or other material employed, are so pressed together that close intimacy of contact of the particles makes the resistance tolerably independent of the current, that then the pieces of carbon will not act as a microphone at all.

We have to thank Messrs. W. Atkinson and Lt. B. Atkinson, two of our students, for much assistance rendered us in these experiments.

II. On Polarizing Prisms. $\quad B y$ R. T. GLAzeBrook, M.A., F.R.S., Fellow and Lecturer of Trinity College, Demonstrator in the Cavendish Laboratory, Cambridge*

TN a paper on Nicol's prism (Phil. Mag. vol. x. 1880) 1 I have considered some of the defects of Nicol's prism as a means of producing plane-polarized light." In the present paper I propose to describe a form of polarizing prism free from most of these. For many purposes, one of the great objections to Nicol's prism is the lateral displacement produced by it in the image of an object viewed through it. If we p'ace a Nicol before the object-glass of a telescope, on turning the Nicol round its axis the image moves across the field. This has been remedied somewhat by cutting prisms with their ends at right angles to their length, and making the angle between the normal to the face on which the incident light falls and the plane of Canada balsam such that the ordinary ray is totally reflected there while the extraordinary ray passes through. But this is not entirely successful; for let $\mathrm{A} B \mathrm{CD}$ (fig. 1) be a section of such a prism by a plane parallel to the edge $\mathrm{BC}$ and at right angles to the Canada balsam. 1883.

* Communicated by the Physical Society of London; read 14th April, 
Tet $P Q$ be the trace of the balsam. In an ordinary Nicol's prism $A B$ would be inclined at about $74^{\circ}$ to $A D$, and $P Q$

Fig. 1.

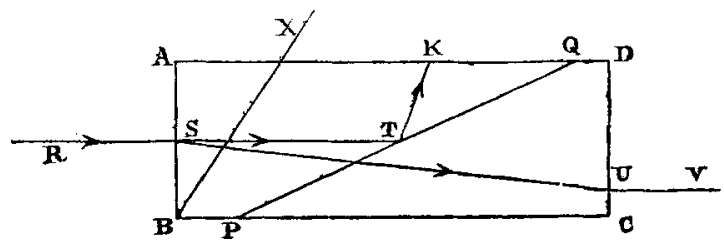

would be at right angles to $A B, A D$ and $B C$ being parallel to edges of the rhomb of spar. In the case now being considered, $\mathrm{AB}$ is at right angles to $\mathrm{BC}, \mathrm{B} \mathrm{C}$ being still parallel to a rhombic edge.

Consider a ray $\mathrm{R} S$ incident normally on A B. The ordinury ray $\mathrm{ST}$ enters the spar without deviation, but is reflected by the balsam at $\mathrm{T}$ in direction $\mathrm{TK}$; the extraordinary ray is refracted at the face $\mathrm{AB}$ in direction $\mathrm{TU}$, and turned from its original path in virtue of the extraordinary refraction. It emerges along $\mathrm{U} V$ parallel to its original direction, but displaced to one side, so that the extraordinary image of the object seen is displaced to one side by the passage of the light through the spar.

In the prism considered in fig. 1, the optic axis lies in the plane of the paper, making an angle of $57^{\circ} 30$ with B C.

Suppose now we cut a rectangular parallelepipedon from a piece of spar, in such a way that two of its faces are at right angles to the optic axis while the other four are parallel to it.

Let A B C D (fig. 2) be a section of the solid by a plane also at right angles to the optic axis, and therefore parallel to two faces and at right angles to the other four; and suppose that

Fig. 2.

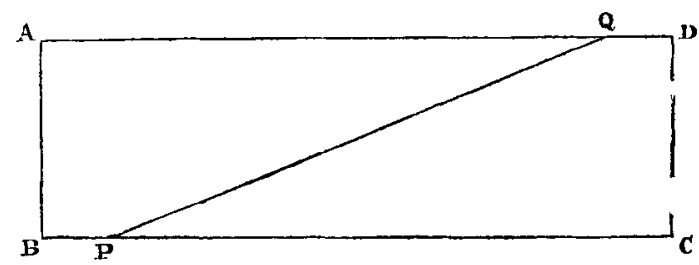

$B C$ is about three times $A B$. Let $P Q$ be inclined at about $20^{\circ}$ to $\mathrm{BC}$, and suppose the prism cut in two by a plane at right angles to the paper and passing through to $P Q$. Then let the faces of section be polished, and cemented together with Canada basam. The optic axis will be at right 
angles to the plane of the paper, and the section of the wavesurface by that plane will be two circles of radii $A$ and $C$, these being the ordinary and extraordinary wave-velocities respectively. Hence a ray falling on the face A B in any direction in the plane of the paper will be divided into two, which will both undergo ordinary refraction, so that if the incident ray be normal to the face $A B$, the extraordinary and ordinary rays in the prism will coincide in direction, both being normal to the same face. The extraordinary ray is not deviated by the refraction; so that no lateral displacement of the extraordinary image is produced by the prism. The ordinary ray is incident at about $70^{\circ}$ on the face $\mathrm{PQ}$; it is therefore totally reflected, and the emergent light is planepolarized. The prism differs from one described by Prof. $\mathbf{S}$. P. Thompson (Phil. Mag. Nov. 1881) only in the fact that its ends are normal to its length instead of being inclined obliquely to it. But this form of prism has other and more important advantages.

Let $\mathrm{OM}, \mathrm{ON}$ (fig. 3) be two extraordinary wave-normals, and $O A$ the optic axis. Pass a plane MOA through OM and OA, and in this plane draw $O P$ at right angles to $\mathrm{OM}$; then $\mathrm{OP}$ is the direction of vibration in the wave which travels along OM. Similarly, if N O A be a plane through $O N$ and $O A$, and $O Q$ a line in it at right angles to $O \mathrm{~N}$, $O Q$ is the direction of vibration for the wave along $\mathrm{ON}$; and it may happen, clearly, that $O P$ and $O Q$ are inclined to one another at a large angle even when $O M$ and $O N$ are close together. Suppose, then, that the extraordinary pencil of wave-normals

Fig. 3.

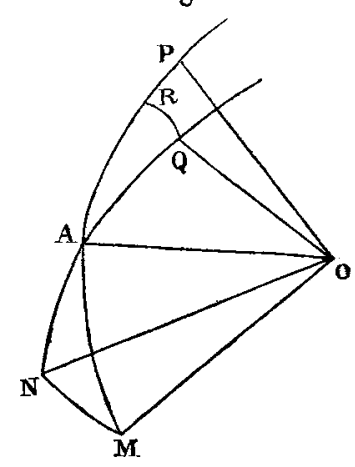
which is traversing the spar is slightly conical, and that $O N$, $O M$ are two of the wave-normals; the planes of polarization are inclined to each other at an angle equal to $\mathrm{POQ}$; and this may be considerable. Or, again, suppose that we have a polarized pencil of parallel wave-normals incident on the prism. We determine the position of their plane of polarization by turning the prism until no light passes through. Suppose that, when this is the case, the incident light is parallel to $O M$. Now let the plane of polarization of the incident light be rotated, and suppose we wish to measure this rotation; we turn the prism until the light is again quenched. Theoretically the axis round which the prism bas been turned should be 
parallel to $0 \mathrm{M}$. In practice it is difficult to ensure this; and in general the direction of the wave-normal relatively to the optic axis will be changed, and may now be $\mathrm{ON}$ say. But since the planes of polarization of the waves along $O M$ and $\mathrm{ON}$ are different, the angle through which the prism has been turned will not be the angle through which the plane of polarization of the incident light has moved.

Now Nicol's prism is so cut that the angle between the planes of polarization of two waves inclined to each other at but a small angle as they traverse the crystal is considerable. If, then, a slightly conical pencil traverse the prism, the angles between the planes of polarization of the different waves are considerable; or if a parallel pencil traverse the prism inclined at but a small angle to the axis of rotation, and the plane of polarization of this beam be rotated, that rotation will differ considerably from the angle through which the prism has to be turned to reestablish blackness.

In our figure the wave along $\mathrm{OM}$ is polarized in a plane at right angles to $O P$, that along $O N$ in a plane at right angles to $O Q$. Consider now a conical pencil of wave-normals in air: it is clearly impossible for it to be plane-polarized, if by plane polarization wo mean that the directions of vibration are parallel to the same line; for we cannot have a series of lines touching a sphere all parallel to the same line. Such a pencil, however, may be said to be most nearly plane-polarized when all the directions of vibration are parallel to the same plane; and this plane will be that which passes through the axis of the pencil and the direction of vibration for the wave-normal which coincides with the axis. For if this be the case, the whole of the pencil can pass unaltered either as an ordinary or extraordinary wave through a piece of spar on which its axis falls normally, provided that the optic axis of the spar be respectively either at right angles to or parallel to the plane in question. Using "plane polarization" in this sense, we proceed to consider when a conical pencil of given vertical angle travelling in a piece of uniaxal crystal is most nearly plane-polarized.

Now let $O M$ (fig. 3 ) be the axis of the pencil, and $O P$ the direction of vibration for the light travelling along $O M$, and let $\mathrm{ON}$ be any other wave-normal. According to the above statement, the conical pencil will be most nearly plane-polarized if the vibration travelling along $O N$ is parallel to the plane POM. If, however, the pencil be travelling in a crystal, it is clearly impossible in general for the displacement along $O N$ to be parallel to this plane. For let $O A$ be the optic axis; OA lies in the plane $\mathbb{M} O \mathrm{P}$. Pass a plane through 
$O A, O N$, and in it draw $O Q$ at right angles to $O N ; O Q$ is the direction of displacement which travels along $O N$, and $O Q$ is not parallel to the plane $P O M$.

We can resolve the displacement along $O Q$ into two, in and perpendicular to the plane POM. The light then will be most nearly plane-polarized when the average intensity of the vibrations normal to this plane is least; and it remains to find the condition for this.

In fig. 3 let $\mathrm{QR}$ be perpendicular to $\mathrm{AP}$. Let $\mathrm{AM}=\alpha$, $\mathrm{NM}=\beta, A N=\theta, A M N=\phi$. Let $\rho$ be the amplitude of the displacement along $O Q$. The displacement normal to the plane PAM is $\rho \sin Q R$, and the intensity of the wave is proportional to $\rho^{2} \sin ^{2} Q R$.

We are to consider a bollow conical pencil with $O M$ as axis. An element of such a pencil at $N$ will be $\sin \beta d \phi$; and the total energy in the pencil, so far as it depends on the displacement normal to the plane PAM, is

Now

$$
\begin{aligned}
& 2 \int_{0}^{\pi} \rho^{2} \sin \beta \sin ^{2} \mathrm{QR} d \phi . \\
& \mathrm{AQ}=\frac{\pi}{2}-\theta, \\
& \sin \mathrm{QK}=\sin \mathrm{AQ} \sin \mathrm{RAQ} \\
&=\cos \theta \sin \mathrm{NAM}, \\
& \sin \mathrm{NAM}=\frac{\sin \phi \sin \beta}{\sin \theta} ; \\
& \therefore \sin \mathrm{QR}=\cot \theta \sin \phi \sin \beta . \quad . \quad . \quad . \quad . \\
& \cos \theta=\cos \alpha \cos \beta+\sin \alpha \sin \beta \cos \phi . \quad . \quad .
\end{aligned}
$$

Also

Substituting in the value of $\sin Q R$, we find for the energy required the expression

$$
2 \rho^{2} \sin ^{3} \beta \int_{0}^{\pi} \frac{\sin ^{2} \phi(\cos \alpha \cos \beta+\sin \alpha \sin \beta \cos \phi)^{2} d \phi}{1-(\cos \alpha \cos \beta+\sin \alpha \sin \beta \cos \phi)^{2}} \text {. }
$$

And we require to evaluate this integral.

Let

Then

$$
\cos \alpha \cos \beta=a, \quad \sin \alpha \sin \beta=b .
$$

$$
\begin{aligned}
& \int_{0}^{\pi} \frac{\sin ^{2} \phi(\cos \alpha \cos \beta+\sin \alpha \sin \beta \cos \phi)^{2} d \phi}{1-(\cos \alpha \cos \beta+\sin \alpha \sin \beta \cos \phi)^{2}} \\
& =\int_{0}^{\pi} \frac{\sin ^{2} \phi(a+b \cos \phi)^{2} d \phi}{1-(a+b \cos \phi)^{2}} \\
& =\int_{0}^{\pi} \frac{\sin ^{2} \phi}{1-(a+b \cos \phi)^{2}} d \phi-\int_{0}^{\pi} \sin ^{2} \phi d \phi \text {. }
\end{aligned}
$$


The first term

But

$$
=\frac{1}{2} \int_{0}^{\pi}\left\{\frac{\sin ^{2} \phi}{1-(a+b \cos \phi)}+\frac{\sin ^{2} \phi}{1+(a+b \cos \phi)}\right\} d \phi .
$$

$$
\begin{aligned}
& \int_{0}^{\pi} \frac{\sin ^{2} \phi d \phi}{c+d \cos \phi}=\int_{0}^{\pi} \frac{\left(1-\cos ^{2} \phi\right) d \phi}{c+d \cos \phi} \\
& =\int_{0}^{\pi}\left\{\frac{c}{d^{2}}-\frac{\cos \phi}{d}-\frac{c^{2}-d^{2}}{d^{2}(c+d \cos \phi)}\right\} d \phi \\
& =\frac{\pi c}{d^{2}}-\frac{c^{2}-d^{2}}{d^{2}} \frac{2}{\sqrt{c^{2}-d^{2}}} \frac{\pi}{2} \\
& \quad \text { if } c \text { is }>d \\
& =\frac{\pi}{d^{2}}\left\{c-\sqrt{c^{2}-d^{2}}\right\} .
\end{aligned}
$$

Hence

$$
\int_{0}^{\pi} \frac{\sin ^{2} \phi}{1-a-b \cos \phi} d \phi=\frac{\pi}{b^{2}}\left\{1-a-\sqrt{\left(1-2 a+a^{2}-b^{2}\right)}\right\} ;
$$

for we can easily show that $c$ is $>d$ in this case. And

$$
\int_{0}^{\pi} \frac{\sin ^{2} \phi}{1+a+b \cos \phi} d \phi=\frac{\pi}{b^{2}}\left\{1+a-\sqrt{\left(1+2 a+a^{2}-b^{2}\right)}\right\} .
$$

And the required integral is

$\frac{\pi}{2 b^{2}}\left\{2-\sqrt{\left(1-2 a+a^{2}-b^{2}\right)-} \sqrt{\left(1+2 a+a^{2}-b^{2}\right)-b^{2}}\right\}$.

But

$$
a^{2}-b^{2}=\cos ^{2} \alpha \cos ^{2} \beta-\sin ^{2} \alpha \sin ^{2} \beta=\cos ^{2} \alpha+\cos ^{2} \beta-1 .
$$

Hence, since the positive sign is to be attached to the roots, we have, if $\beta$ be $<\alpha$,

Intensity required

$$
\begin{aligned}
& =\frac{\pi \rho^{2} \sin \beta}{\sin ^{2} \alpha}\left\{2-(\cos \beta-\cos \alpha)-(\cos \beta+\cos \alpha)-\sin ^{2} \beta \sin ^{2} \alpha\right\} \\
& =\pi \rho^{2} \sin \beta(1-\cos \beta)\left\{\frac{2}{\sin ^{2} \alpha}-(1+\cos \beta)\right\} \\
& =4 \pi \rho^{2} \sin \beta \sin ^{2} \frac{\beta}{2}\left\{\operatorname{cosec}^{2} \alpha-\cos ^{2} \frac{\beta}{2}\right\} . . . . . . .
\end{aligned}
$$


And if $\alpha$ be $<\beta$,

Intensity

$=\pi \rho^{2} \sin \beta\left\{\frac{2}{1+\cos \alpha}-\sin ^{2} \beta\right\}=\pi \rho^{2} \sin \beta\left\{\sec ^{2} \frac{\alpha}{2}-\sin ^{2} \beta\right\}$.

In the first case the intensity is clearly least when $\alpha$ is $\frac{\pi}{2}$, its value then being

$$
\pi \rho^{2} \sin \beta(1-\cos \beta)^{2} \text {; }
$$

and in the second case it is least when $\alpha$ is 0 , and its value is

$$
\pi \rho^{2} \sin \beta \cos ^{2} \beta \text {. }
$$

This second minimum will be greater than the other if

$$
\begin{array}{ll}
\text { i. e. if } & \cos \beta \text { is }>\frac{1}{2}, \\
\text { i. e. if } & \quad \beta \text { is }<60^{\circ} .
\end{array}
$$

If, then, a conical pencil whose semi- vertical angle is less than $60^{\circ}$ be passing through the spar, the pencil will be most nearly plane-polarized if the axis of the pencil is at right angles to that of the spar.

Now if the axis of a conical pencil pass normally through a prism cut as already described, it will be at right angles to the optic axis; and hence the pencil, if its semi- vertical angle be less than $60^{\circ}$, will be more nearly plane-polarized than it would be if the axis occupied any other position. This constitutes a second advantage in favour of the new prism.

Again, suppose we have a parallel pencil of wave-normals in direction $O N$, and that the axis round which the prism rotates is OX (fig. 4). In our observations we suppose that these two coincide, and work as if the plane of polarization of the emergent light coincided with that of light travelling along $O X$, thus introducing an error. The amount of this error will depend of course partly on the angle NX ( $\beta$ say), and partly on the angle NXA ( $\phi$ say), OA being the optic axis. If we know $\beta$ and $\phi$ we can calculate the error, and could determine the value to be given to XA or $\alpha$ to make it the least possible.

But in practice $\phi$ may be anything between 0 and $2 \pi$, and $\beta$ anything between 0 and a not very large angle $\beta_{1}$; and the question arises, what value must we assign to $\alpha$ in order that the error produced by any chance values of $\beta$ and $\phi$ may most probably be as small as possible? To answer this we require to determine, between these limits for $\beta$ and $\phi$, the 
mean of the values, irrespective of sign, of the amplitudes of the vibrations normal to the plane OAX.

Let $O Q$ be the direction of vibration for the wave-normal $O N$, and $Q R$ perpendicular on the plane AX. Then the displacement normal to the plane $\mathrm{AOX}$ is $\rho \sin \mathrm{QR}$; and, with the same notation as before, the average displacement

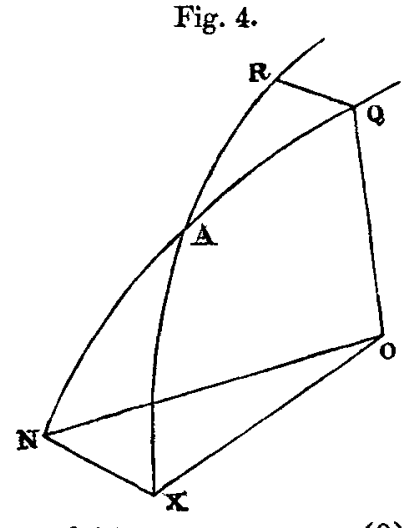

$$
\begin{aligned}
& =\frac{\rho}{2 \pi} \int \sin \beta \sin \phi \cot \theta d \phi . \quad . \quad . \quad . \\
& \theta=\sin \alpha \sin \beta \sin \phi d \phi . \\
& \text { displacement } \\
& =\frac{\rho}{2 \pi \sin \alpha} \int \cos \theta d \theta . . \\
& \text {. . . . . . }
\end{aligned}
$$

Also $\quad \sin \theta d \theta=\sin \alpha \sin \beta \sin \phi d \phi$.

Hence average displacement

We must consider now the limits between which the integral is to be taken.

Describe a circle (fig. 5) passing through $\mathrm{N}$ with $\mathrm{X}$ as centre, and cutting $A X$ in $L$ and $M$, and if possible let $N_{1}$ be a point on this circle such that

$$
\mathrm{N}_{1} \mathrm{~A}=\frac{\pi}{2}
$$

Suppose we treat displacements to the right of $\mathrm{AX}$ as positive. When $\mathrm{N}$ coincides with $\mathrm{L}$,

$$
\theta=\alpha-\beta \text {. }
$$

Fig. 5.

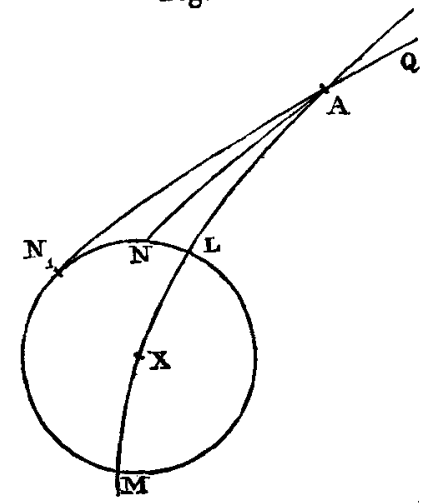

If $N$ lies between $L$ and $N_{1}, Q$ is to the right of $A X$, as in figure, and the displacement is positive. We must therefore integrate for $\theta$ from $\alpha-\beta$ to $\frac{\pi}{2}$. But if $N$ lie between $N_{1}$ and $\mathrm{M}, \mathrm{Q}$ will be to the left of $\mathrm{AX}$, and the displacement will be negative. Thus, to get the whole average displacement, irrespective of sign, we have to subtract the value of the integral from $\frac{\pi}{2}$ to $\alpha+\beta$. If, however, $\alpha+\beta$ is $<\frac{\pi}{2}$, no position such as $\mathrm{N}_{1}$ can be found, and we have to integrate straight from 
$\alpha-\beta$ to $\alpha+\beta$. The same is true for positions of $N$ on the other side of $\mathrm{AX}$.

Hence, in the first case, the average displacement normal to the plane is

$$
\begin{aligned}
& \frac{2 \rho}{2 \pi \sin \alpha}\left\{\int_{\alpha-\beta}^{\frac{\pi}{2}} \cos \theta d \theta-\int_{\frac{\pi}{2}}^{\alpha+\beta} \cos \theta d \theta\right\} \\
& \quad=\frac{\rho}{\pi \sin \alpha}\{1-\sin (\alpha-\beta)+1-\sin (\alpha+\beta)\} \\
& \quad=\frac{2 \rho}{\pi}\left\{\frac{1}{\sin \alpha}-\cos \beta\right\} \\
& \quad=\frac{2 \rho}{\pi}\{\operatorname{cosec} \alpha-\cos \beta\} . . . . .
\end{aligned}
$$

And in the second case it is

$$
\begin{aligned}
\frac{2 \rho^{\prime}}{2 \pi \sin \alpha} \int_{\alpha-\beta}^{\alpha+\beta} \cos \theta d \theta & =\frac{\rho}{\pi \sin \alpha}\{\sin (\alpha+\beta)-\sin (\alpha-\beta)\} \\
& =\frac{2 \rho}{\pi} \cot \alpha \sin \beta .
\end{aligned}
$$

The first is clearly least when $\alpha$ is $\frac{\pi}{2}$; the second decreases as $a$ increases, but has no minimum ; for after a time we should reach a point at which $\alpha+\beta$ became equal to $\frac{\pi}{2}$, and then the limits would require changing : for this value, of course, the two integrals are the same.

Thus the average displacement normal to the plane OAX is least when $O X$ is at right angles to the optic axis, and hence the average error in the position of the plane of polarization is least also. The average displacement just calculated is of course that for a given value of $\beta$. If we require the average for any value of $\beta$ between 0 and $\beta_{1}$, we must multiply our expressions (11) and (12) by $d \beta$, and, integrating from 0 to $\beta_{1}$, divide the result by $\beta_{1}$.

To show the difference in this respect between the new prism and Nicol's, let us calculate the displacement normal to the plane $A O X$ in the two cases, supposing the value of $\beta$ is $5^{\circ}$. In Nicol's prism, $\alpha=63^{\circ} 30^{\prime}$; so that $\alpha+\beta$ is less than $\frac{\pi}{2}$, and the second formula (12) must be taken. The ratio of the two displacements is therefore

$$
\frac{\cot 63^{\circ} 30^{\prime} \sin \beta}{1-\cos \beta}=\cot 63^{\circ} 30^{\prime} \cot \frac{\beta}{2}
$$


and substituting the value $\beta=5^{\circ}$, this comes to $434: 39$, or about 11 to 1 . Thus the average error in the position of the plane of polarization as determined by the new prism will be about one eleventh of that which would be produced by the same errors of adjustment with a Nicol's prism; while the amount of light polarized out of the proper plane will be less than one per cent. of that which would be produced by a Nicol.

Again, suppose the prism is turned through an angle $\omega$ about OX (fig. 6), and let us inquire what is the angle through which the plane of polarization of the emergent light is rotated. Let $\mathrm{OA}^{\prime}$ be the new position of the optic axis. Join $\mathrm{MA}, \mathrm{MA}^{\prime}$, and in them take points $\mathrm{P}, \mathrm{P}^{\prime}$ such that $\mathrm{MP}=\mathrm{MP}^{\prime}=\frac{\pi}{2}, \quad \mathrm{OP}, O \mathrm{P}^{\prime}$ Fig. 6.

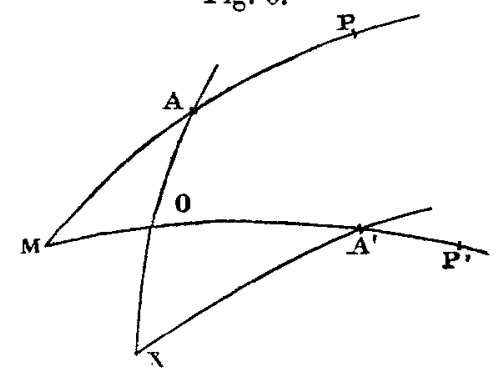

are the directions of vibration for the waves travelling along $\mathrm{OM}$ in the two positions of the prism respectively. The angle through which the plane of polarization has been turned is $\mathrm{P}^{\prime}$ or $\mathrm{PM} \mathrm{P}^{\prime}$, that through which the prism has been turned is $\mathrm{A} \mathrm{X} \mathrm{A}^{\prime}$; and we require to investigate the conditions under which the average difference between these two for all possible positions of $M$ within a certain distance, $\beta_{1}$ say, of $X$.

Now we have seen already that if the axis of rotation be at right angles to the optic axis, the average error produced in the determination of the position of the plane of polarization for each of the two positions of the prism will be a minimum; and hence it follows that the average error in the angle between these two positions is a minimum also.

All these results, of course, hold only for the position of the plane of polarization of the light when in the crystal, and will be modified by the refraction that takes place as the waves emerge into the air. But since the ends of the prism are normal to its length, for all the waves considered the incidence is very nearly direct, and the change produced by refraction in the position of the plane of polarization is very small indeed.

Thus a prism cut as described possesses the following advantages over Nicol's prism:-

1. There is no lateral dispacement in the apparent position of an object viewed through it.

2. A conical pencil whose axis passes directly through is more nearly plane-polarized than would be the case if the axis of the prism were related to that of the spar in any other manner. 
3 . If the direction of the wave-normal within the prism does not quite coincide with the axis of rotation, the average error in the position of the plane of polarization is less than for any other method of cutting.

I hope shortly to have some prisms cut by Mr. Hilger in this manner, and to test by means of them the theoretical conclusions arrived at in the paper.

\section{Note added April 26th.}

If the plane of section $\mathrm{PQ}$ be inclined to $\mathrm{BC}$ at an angle of $20^{\circ}$, as in fig. 2, the angular aperture of the field will be small, only about $10^{\circ}$, and it will be necessary that all the light traversing the prism should be very nearly parallel to BC. The aperture may be increased up to about $20^{\circ}$ by lengthening the prism considerably and decreasing the angle between $P Q$ and $B C$. If this be reduced to $11^{\circ}$, the aperture will have its maximum value of $22^{\circ}$.

The aperture may be somewhat increased, and the length of the prism shortened, by using as the separating medium balsam of copaiba, as was suggested at the meeting of the Physical Society at which this paper was read.

The mean index of refraction for this substance is about 1.52, as determined by Brewster. The angle of total reflexion therefore for the ordinary ray is $\sin ^{-1}(1 \cdot 52 / 1 \cdot 66)$, or about $66^{\circ}$, while for Canada balsam this angle is about $68^{\circ}$. The possible aperture, using the balsam of copaiba, thus is about $24^{\circ}$.

Professor Thomson's prism, mentioned already, will have a wider field. But it must be remembered that the new prism was not designed for microscopic work, but to obviate the displacement in the image referred to at the commencement of the paper, and to produce a field in which the plane-polarization should be as nearly as possible complete.

\section{Notices respecting New Books.}

Physical Optics (Text-books of Science). By R. T. GLazeBroor, M.A. \&o. (London: Longmans, Green \& Oo. Pp. 434+xiv.)

THIS is an important elementary work, chiefly on "physical optics ;" 1 but it contains also much of what is commonly termed "geometrical optics." It is on the whole most lucidly written, and gives a capital idea of the subject to those who wish to grasp a sound knowledge of it without going into the higher mathematical analysis. The work purports to be adapted for "artisans and students in public and science schools : " it is, however, partly from the general difficulty of parts of the subject and partly from the 